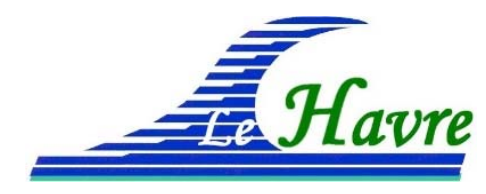

XVI ${ }^{\text {èmes }}$ Journées Nationales Génie Côtier - Génie Civil
Le Havre, 2020

DOI:10.5150/jngcgc.2020.032 C Editions Paralia CFL

disponible en ligne - http://www.paralia.fr - available online

\title{
Modeling the initiation of underwater granular avalanches: an Eulerian approach
}

\author{
Eduard PUIG MONTELLÀ̀ ${ }^{1}$, Julien CHAUCHAT ${ }^{1}$, \\ Cyrille BONAMY ${ }^{1}$, Tian-Jian HSU ${ }^{2}$
}

\author{
1. University of Grenoble Alpes, LEGI, G-INP, CNRS, 38000 Grenoble, France. \\ eduard.puig-montella@univ-grenoble-alpes.fr \\ 2. Civil and Environmental Engineering, Center for Applied Coastal Research, \\ University of Delaware, Newark, DE 19711, USA.
}

\begin{abstract}
:
In this work, the two-phase flow model SedFoam is developed to reproduce the initiation of underwater granular avalanches. In agreement with previous results, the behavior of a layer of granular material fully immersed in a fluid flowing down an inclined slope is strongly influenced by the initial volume fraction. Loose granular packings accelerate rapidly, whereas dense granular packings exhibit a time delay before flowing. Indeed, shear stresses exerted by the fluid flow on the granular bed not only induce a motion on the tangent component but also a motion in the wall-normal direction. Such behavior is explained by a combination of the dilatancy angle and pore pressure feedback on the grains. Dilation mechanism increases the volume fraction triggering significant pore pressures inside the granular layer. Additionally, fluid is sucked into the pore space pressing the solid particles together, leading to increased normal stress and the frictional forces. Likewise, contraction results in an increment of the pore pressure and a reduction of the shear strength. The dynamics of underwater avalanches captured by the numerical model accounting for the dilatant-contractant behavior are qualitatively validated with available experimental data. This work is a first step toward incorporating these mechanisms in the Eulerian framework. This a major step in the development of the model that will allow to tackle more complex problems in the future, such as piping, granular collapse or mine burial.
\end{abstract}

\section{Keywords:}

Eulerian approach, Granular flows, Fluid-solid phase coupling, Dilatancy.

\section{Introduction}

Modeling and predicting granular flows are of major interest to accurately describe landslides, submarines avalanches and sediment transport. Grain-fluid mixtures exhibit a strong coupling between the fluid and solid phases that can significantly modify the mixture behavior. Indeed, the motion of the solid grains modifies the boundary conditions of the fluid potentially altering the global response. In this report, several simulations are 


\section{Thème 2 - Dynamique sédimentaire}

conducted with the open-source software SedFoam, a two-phase flow solver frequently used for sediment transport applications (CHAUCHAT et al., 2017; MATHIEU et al., 2018). The solver is available for download on GitHub: https://github.com/SedFoam/sedfoam. In this approach, the sediment phase is treated as continuum where constitutive relations are needed to model inter-particle stresses. The current version of SedFoam computes such stresses with two implemented models: the kinetic theory of granular flows and the dense granular flow $\mu(I)$-rheology. The present benchmark focus on physical problems where the dense granular flow $\mu(I)$-rheology is considered.

We consider an avalanche of a solid phase flowing down a planar slope as illustrated in figure 1a. Initially, sediment is deposited by gravity forming a granular layer. Then, the system is rotated and gravitational forces accelerate the mixture. According to previous results (PAILHA et al., 2008; PAILHA \& POULIQUEN, 2009; IVERSON, 2012), the motion of dense granular layers (see figure 1b) do not start immediately. Negative pore fluid pressures develop in the granular layer due to the fluid sucked into the solid phase. Although such mechanism leads to dilation of the granular medium, particles are pressed together hindering the motion of the granular medium. On the contrary, loose packings are characterize by a rapid acceleration in which velocity increases linearly from the beginning. In this case, fluid flows out the pore space enhancing the compaction of the granular layer.
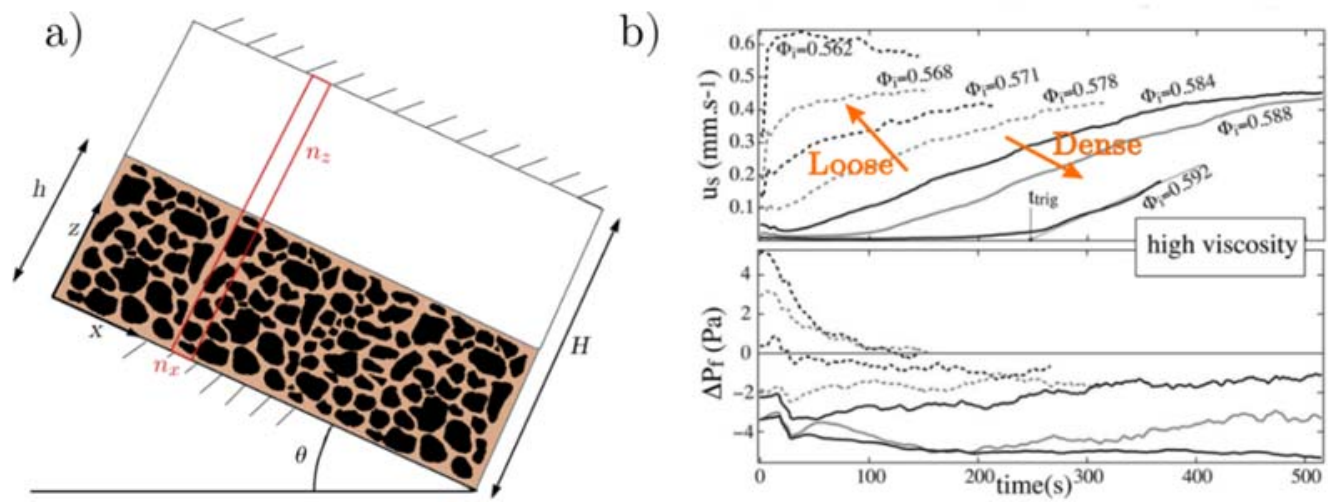

Figure 1. a) Schematic section of the avalanche made up of a solid-fluid mixture flowing down a uniform slope. b) Experimental surface velocity $\left(u^{s}\right)$ and pore pressure $\left(\Delta P^{f}\right)$ for different initial volume fractions ( $\phi)$ extracted from PAILHA et al., 2008.

In this work, numerical results are contrasted with the analytical solution obtained after assuming the $\mu(I)$-rheology when the granular flow is fully developed. In addition to the theoretical results, SedFoam simulations based on the experimental setup of PAILHA et al. (2008) show the ability of the model to capture the initiation of underwater avalanches for packings at the critical state. Finally, numerical simulations accounting for the 


\section{XVIèmes Journées Nationales Génie Côtier - Génie Civil \\ Le Havre 2020}

dilatant-contractant behavior evidence the initial dynamics of dense packings are qualitatively well reproduced by the numerical model.

Two scenarios are distinguished in our numerical simulations:

- Case D - A dry avalanche. Typically, an air-solid mixture avalanche.

- Case W - A submarine avalanche in which the fluid phase has a high viscosity.

The setup and main variables are presented in figure 1 and table 1:

Table 1. Physical parameters used in the numerical simulations.

\begin{tabular}{lccc}
\hline Parameter & SI unit & Dry avalanche $(\boldsymbol{D})$ & Wet avalanche $(\boldsymbol{W})$ \\
\hline Solid phase density $\rho^{p}$ & $\mathrm{~kg} / \mathrm{m}^{3}$ & 2650.0 & 2500.0 \\
Fluid phase density $\rho^{f}$ & $\mathrm{~kg} / \mathrm{m}^{3}$ & 1.0 & 1041.0 \\
Fluid viscosity $v^{p}$ & $\mathrm{~m}^{2} / \mathrm{s}$ & $1.0 \cdot 10^{-3}$ & $9.2 \cdot 10^{-5}$ \\
Particle diameter $d$ & $\mathrm{~m}$ & $500 \cdot 10^{-6}$ & $160 \cdot 10^{-6}$ \\
Plane slope $\theta$ & - & $26^{o}$ & $25^{\circ}$ \\
Bed thickness $h$ & $m$ & 0.0125 & 0.0049 \\
\hline
\end{tabular}

\section{Numerical model}

The two-phase flow model presented in this work was introduced by CHAUCHAT et al. (2017) and is based on an Eulerian description of the solid and fluid phases. We refer the reader to CHAUCHAT et al. (2017) for more details on the mathematical formulation. Regarding the solid phase, two different intergranular stress models are currently implemented: the kinetic theory of granular flows and the dense granular flow $\mu(I)-$ rheology. In this work, we focus our attention on particle stresses obtained with the $\mu(I)$ rheology.

\subsection{Dense granular $\mu(I)$-rheology}

Although several works have studied the role of different parameters that trigger and describe the motion of granular mixtures (MIDI, 2004; POULIQUEN \& FORTERRE, 2009), continuum granular flow rheology remains an open problem. Despite these challenges, the $\mu(I)$-rheology is one of the most popular model to reproduce dense granular flows. The model establishes that the ratio of shear, $\tau^{p}$, to normal stresses, $p^{p}$, hereafter denoted as $\mu$, is a unique function of a dimensionless number I (DA CRUZ, 2004):

$\mu(I)=\frac{\tau^{p}}{p^{p}}$

where $I=\frac{t_{\text {micro }}}{t_{\text {macro }}}=\frac{\dot{\gamma} d}{\sqrt{p^{p} / \rho^{p}}}$ is the inertial dimensionless number given by the ratio of two time scales: the time scale of rearrangement $t_{\text {micro }}=\frac{d}{\sqrt{p^{p} / \rho^{p}}}$ and the macroscopic time scalet $t_{\text {macro }}=\frac{1}{\gamma}$. Here, $d$ stands for the particle diameter and $\gamma=\frac{\partial u^{p}}{\partial z}$ is the shear rate. 


\section{Thème 2 - Dynamique sédimentaire}

Following MIDI (2004) and BOYER et al. (2011), the friction coefficient can be expressed as:

$\mu(I)=\mu_{s}+\frac{\mu_{2}-\mu_{s}}{\frac{I_{O}}{I}+1}$

Where the friction coefficient $\mu(I)$ tends to a minimum value $\left(\mu_{s}\right.$ when $I \rightarrow 0$ and converges to a maximum value $\left(\mu_{2}\right)$ at high shear rate.

Viscous forces become dominant if the Stokes number $\left(S t=\gamma^{\prime} \rho^{p} d^{2} / \eta^{f}\right.$, where $\eta^{f}$ is the fluid dynamic viscosity) is significantly small. Under such circumstances, the rheology of the system is governed by the dimensionless viscous number:

$I_{v}=\frac{\gamma \eta^{f}}{p^{p}}$

\subsection{Dilatancy model}

Dilatancy is an inherent characteristic of granular materials that plays a key role in the rheology of dense granular flows. Dilatancy describes the changes in the solid volume fraction subjected to shear deformations. Additionally, dilatancy modifies the pore pressure, and subsequently, the frictional force between particles. This study aims to develop a two-phase model that can properly reflect the effects of shear dilatancy.

The momentum governing equations for the solid phase in the Eulerian system are:

- In the stream-wise ( $x$-axis) direction:

$$
\frac{d u^{p}}{d t}=\frac{\rho^{p}-\rho^{f}}{\rho^{p}} g \cdot \sin \theta+C_{D}\left(u^{f}-u^{p}\right)+\frac{\partial}{\rho^{p} \partial z}\left(\mu\left(I_{v}\right)+\delta\right) p^{p}
$$

- In the vertical (z-axis) direction:

$$
\frac{d v^{p}}{d t}=\frac{\rho^{p}-\rho^{f}}{\rho^{p}} g \cdot \cos \theta+C_{D}\left(v^{f}-v^{p}\right)-\frac{\partial p^{p}}{\partial z}-\phi \frac{\partial p^{f}}{\partial z}+\frac{\partial}{\partial z}\left(p_{e q} \frac{\mu_{b}(\phi)}{\left|\frac{\partial u^{p}}{\partial z}\right|} \frac{\partial u^{p}}{\partial z}\right)
$$

where $C_{D}$ is the drag coefficient, $\mu_{b}$ is the equivalent of a bulk viscosity and $p_{e q}$ is the equilibrium confining pressure.

Eq. 4 states that under a steady state, the action of gravity is balanced by the contribution of drag and frictional forces. Eq. 4 introduces the concept of dilatancy angle $\delta$, which gives the rate of dilatation (or compaction) of a granular material undergoing through shear deformations. Essentially, the dilatation of a dense packing $(\delta>0)$ comes with an increment of the apparent friction coefficient (see Eq.4) while the compaction of a loose packing $(\delta<0)$ leads to a lower apparent friction. Plus, the dilatancy strength vanishes towards the critical state. Following PAILHA and POULIQUEN (2009), the dilatancy angle is assumed to be linearly proportional to the difference between the current volume fraction and the volume fraction obtained at the steady state $\left(\phi_{e q}\right)$ :

$\delta=K_{1}\left(\phi-\phi_{e q}\right)$

The last term of Eq.5 suggests that the granular layer may experience motion in the $Z$ direction due to the dilatancy effects. 


\section{XVİ̀mes Journées Nationales Génie Côtier - Génie Civil \\ Le Havre 2020}

\subsection{Theoretical solution}

In the stream-wise direction, neglecting the slip velocity between the phases, the momentum conservation equations is reduced to:

$0=\frac{d \tau_{x z}^{p}}{d z}+\frac{d \tau_{x z}^{f}}{d z}+\rho^{m} g \cdot \sin \theta$

where $\rho^{m}$ is the mixture density, $\tau^{p}$ is the particle shear stress and $\tau^{f}$ is the fluid shear stress. By neglecting the fluid shear stress and integrating the momentum equation along the wall-normal direction $z$, the velocity profiles for the inertial and viscous regimes are given, respectively:

$$
\begin{aligned}
& u(z)=\frac{2 I \sqrt{\phi g \cdot \cos \theta}}{3 d}\left(h^{\frac{3}{2}}-(h-z)^{3 / 2}\right) \\
& u(z)=I_{v} \frac{\left(\rho^{p}-\rho^{f}\right) \phi g \cdot \cos \theta}{2 \eta^{f}}\left(h^{2}-(h-z)^{2}\right)
\end{aligned}
$$

\section{Results}

\subsection{Dry avalanche - Case D}

Figure 2 shows the numerical solution for the solid volume fraction, velocity, pressure, $\mu(I)$, shear stress and velocity gradient along the vertical axis. In order to test the accuracy of the model, figure 2 incorporates the theoretical solution derived in section 2.3, hereafter referred as the Bagnold solution. Overall, the numerical results are consistent with the analytical solution since the evolution of the main parameters are well captured by the numerical model. However, some discrepancies arise in terms of velocity profile, frictional viscosity and shear rate. Such differences are more pronounced in the vicinities of the solid-fluid interface.
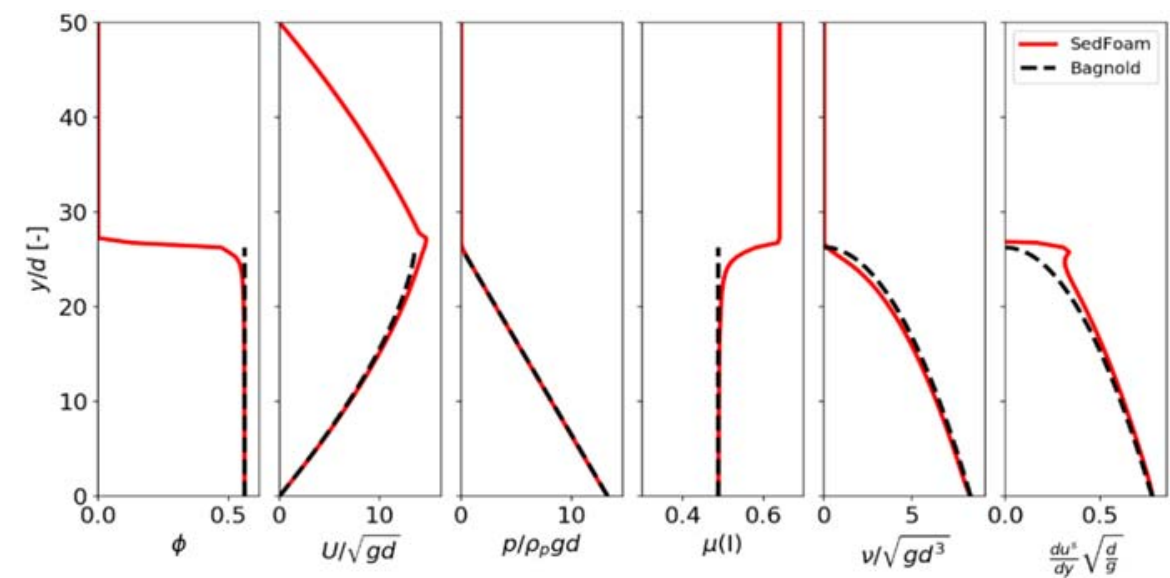

Figure 2. Evolution of a) solid volume fraction, $b$ ) velocity, $c$ ) solid phase pressure, $d$ ) $\mu(I), f)$ frictional viscosity, and $g$ ) shear rate in function of height. Case $D$. 


\section{Thème 2 - Dynamique sédimentaire}

\subsection{Underwater avalanche - Case W}

We now consider an avalanche of a granular bed fully immersed in a water-oil mixture. The physical and geometrical parameters have been adjusted to mimic the experimental setup of PAILHA et al. (2008). The numerical results are contrasted to the analytical solution obtained after assuming the $\mu\left(I_{v}\right)$-rheology (see analytical solution in section 2.3). Additionally, experimental data have been included in the comparison. Figure 3a shows the theoretical surface particle velocity when the granular avalanche is fully developed (Bagnold solution in figure 3a). The numerical simulation does not exactly converge towards the analytical solution as in the dry avalanche. The gap between the curves suggests the analytical approach based on the hypothesis that fluid shear stresses are negligible is probably not an accurate solution under the viscous regime.
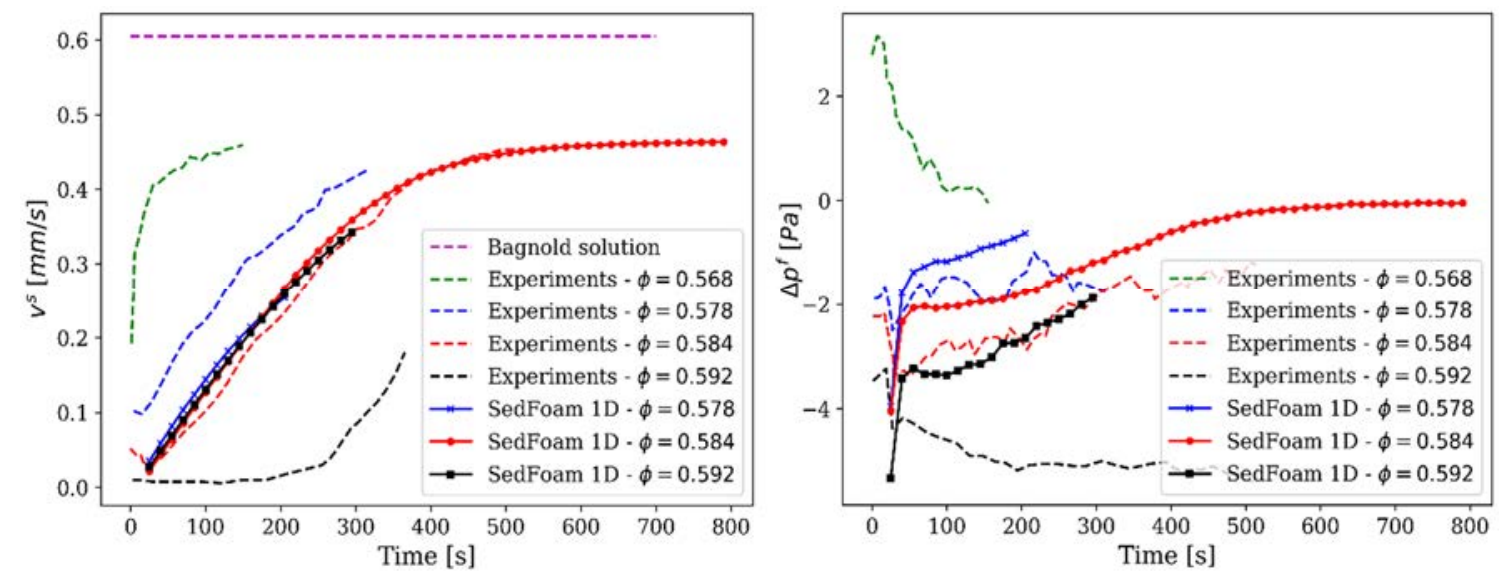

Figure 3. Evolution of a) surface particle velocity and b) pore pressure along time. 1D simulations with different initial volume fractions $(\phi)$ are considered. Case $W$.

In addition to the steady state, we also investigate the behavior of the granular avalanche when the flow starts developing. Figure 3 displays the time evolution of the surface particle velocity and excess of pore pressure. The agreement is correct for both initial and fully developed avalanche.

Numerical simulations for the same initial volume fractions as in the experiments have been performed using SedFoam (see solid lines in figure 3). Numerical results show good agreement with available experimental (PAILHA et al., 2008). At the critical state $(\phi \approx$ 0.580 ) but, unlike the experiments, no sensitivity to the initial volume fraction is revealed. Due to the lack of dilatancy, all the granular packings adopted in this section $(\phi=$ $0.578, \phi=0584$ and $\phi=0.592$ ) behave according to the critical state. The experimental dense granular layer (red dashed curve in figure 3 ) barely flows during the first 5 seconds. Furthermore, the predicted pore pressures agree well with the experimental results: the initiation of the avalanche comes along a negative pore pressure. 


\section{XVİ̀mes Journées Nationales Génie Côtier - Génie Civil \\ Le Havre 2020}

The pressure builds up rapidly after tilting the box, then, the pore pressure progressively increases as the avalanche develops.

\subsection{Dilatancy effects}

Figure 4 is obtained after a 1D simulation where the dilatancy angle was computed following Eq.6. According to figure 4 , the behavior of the dense packing $(\phi=0584)$ is correctly predicted: initially, the granular layer flows at a slow pace. After a few seconds, the avalanche accelerates at a similar pace as the critical state (see parallel paths in figure 4). Despite the qualitative agreement between the curves, more accurate results are needed to fully describe the dilatant-contractant behavior.

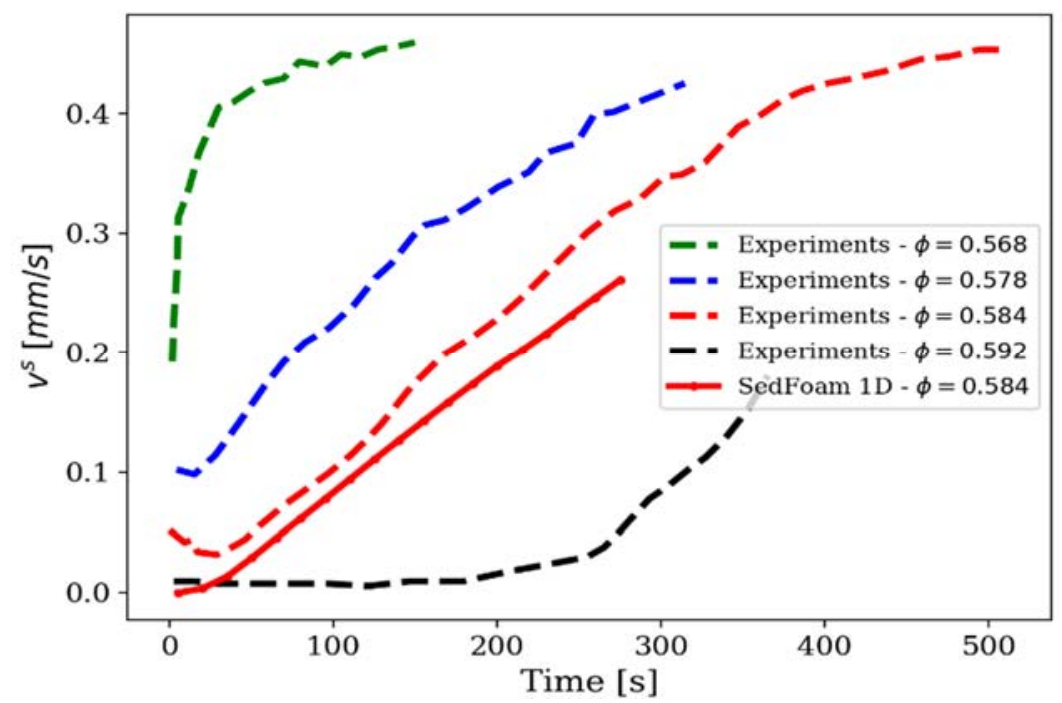

Figure 4. Evolution of a) surface particle velocity and b) pressure with time when the dilatancy model is implemented. Case W.

\section{Conclusions}

In this contribution, a multi-dimensional continuum formulation was proposed. Numerical simulations were compared with experiments and theoretical solutions to illustrate the capability of the model to capture the transient and stationary regimes of dry and wet granular avalanches. Despite the accuracy of SedFoam to describe initial and fully developed granular flows, improvements are needed to mimic the initial deformations and reproduce the strong influence of dilatancy on the dynamics of granular flows. Even though we briefly introduced the recent developments on the dilatancy model, more efforts are devoted to extend our model beyond the critical state. 


\section{Thème 2 - Dynamique sédimentaire}

\section{References}

BOYER F., GUAZZELLI E., POULIQUEN O. (2011). Unifying suspension and granular rheology, Physical Review Letters, Vol. 107, 188301. https://doi.org/10.1103/PhysRevLett.107.188301

CHAUCHAT J., CHENG Z., NAGEL T., BONAMY C., HSU T.-J. (2017). Sedfoam2.0: a 3-d two-phase flow numerical model for sediment transport. Geoscientific Model Development, Vol. 10(12), pp 4367-4392. https://doi.org/10.5194/gmd-10-4367-2017

DA CRUZ F. (2004). Ecoulement de grains secs: Frottement et blocage. PhD thesis, ENPC, 283p. https://pastel.archives-ouvertes.fr/pastel-00000946

IVERSON R.M. (2012). Mechanics of debris flows and rock avalanches. Handbook of Environmental Fluid Dynamics, Vol. 1, pp 591-624. https://doi.org/10.1201/b14241-47

MATHIEU A., NAGEL T., BONAMY C., CHAUCHAT J., CHENG Z., LIU X., HSU T.-J. (2018). Application of a eulerian two-phase flow model to scour processes. in E3S Web of Conferences, River Flow 2018, Vol. 40, 05015, EDP Sciences, 8p. https://doi.org/10.1051/e3sconf/20184005015

MIDI G. (2004). On dense granular flows. The European Physical Journal E, Vol. 14(4), pp 341-365. https://doi.org/10.1140/epje/i2003-10153-0

PAILHA M., NICOLAS M., POULIQUEN O. (2008). Initiation of underwater granular avalanches: influence of the initial volume fraction. Physics of fluids, Vol. 20(11), 111701. https://doi.org/10.1063/1.3013896

PAILHA M., POULIQUEN O. (2009). A two-phase flow description of the initiation of underwater granular avalanches. Journal of Fluid Mechanics, Vol. 633, pp 115-135. https://doi.org/10.1017/S0022112009007460

POULIQUEN O., FORTERRE Y. (2009). A non-local rheology for dense granular flows. Philosophical Transactions of the Royal Society A: Mathematical, Physical and Engineering Sciences, Vol. 367(1909), pp 5091-5107. https://doi.org/10.1098/rsta.2009.0171 